\title{
Effect of an Emotion Regulation TrainingProgram on Mental Well-Being
}

\author{
Sara Leblanc - PhD; Bilge Uzun - PhD; Katere Poureseied - PhD
}

\section{ABSTRACT}

This study aimed to develop and validate a brief emotion regulation training program that improved mental well-being. The program was preventative in nature, designed to build resilience while fostering more satisfying personal and professional relationships in a community sample. It was developed and piloted with 10 graduate students during Study 1 ; forStudy 2 , a community sample of 75 adults were randomly allocated to either a treatment $(n=38)$ or control $(n=37)$ group. In the pilot program, the social validity of the program wasconfirmed, while study 2 demonstrated significant changes in positive coping techniques (i.e.,reappraisal) at one-year follow-up in the treatment group when compared with the control. These findings may have broad implications in the health sector due to the brevity, scalability, and costeffectiveness of this newly designed intervention.

Emotion regulation is a vibrant topic in contemporary psychology. It provides insight into mental functioning and the relative impactcognitive processes have on well-being (Bebko, Franconeri, Ochsner, and Chiao, 2011; Gross, 2013). Emotion regulation is defined as the heterogeneous set of processes an individual implements to modulatetheir emotional experience (Gross, 1998). The ability to effectivelyregulate emotions is a vital skill that has been linked to increased lifesatisfaction (Gross \& John, 2003) and enhanced interpersonal relationships (Koole, 2009). Concordantly, the inability to regulate emotions has severe consequences and has been linked to elevated stress(McLaughlin \& Hatzenbuehler, 2009), anxiety (Cisler \& Olatunji, 2012), and depression (Joormann \& Gotlib 2010) and is a cardinaldiagnostic feature in more than half of all mental disorders (Gross \& Levenson, 1997). Therefore, it appears that preventative psychologicalinterventions are needed due to both the prevalence and the deleterious impact of mental illness. In an attempt to fill this need, a variety of targeted and early intervention programs have been developed, such as Coping with Depression (Cuijpers, Munoz, Clarke, \& Lewinsohn, 2009), Don't Panic (Meulenbeek et al., 2010), and Living Life to the Full (Fledderus, Bohlmeijer, Smit, \& Westerhof, 2011). Although these programs have demonstrated efficacy for both clinical and non-clinical samples (for a review, see Bolier et al., 2013;Sin \& Lyubomirsky, 2009), broad emotion skills training programs that focus on psychoeducation for community samples are still relatively uncommon in large-scale applications. In addition, the pro- grams offered are often not grounded in scientific theory, and usenumerous techniques with an ambiguous psychological base (Kotsou, Nelis, Gregoire, \& Mikolajczak, 2011); moreover, within a research setting, they often have high attrition rates and employ small samplesizes (Baer, 2003). Thus, the construction of the current research wasan attempt to address some of the key limitations existing within the current literature.

To address concerns of a theoretical nature, the current research was grounded in the process model of emotion regulation (Gross, 1998). Inaddition, a control group was employed and a one-year follow-up wasconducted to test enduring intervention effects. Generally speaking, theprogram consisted of three parts: education, activities, and bibliotherapy.

In the current intervention, a variety of cognitive and behavioraltechniques were taught in order to account for individual difference and personal preference. Cognitive techniques included reappraisal (viewing a situation in a different way to change its emotional impact)(John 
\& Gross, 2004), mindfulness, and expressive writing, while behavioral techniques included diaphragmatic breathing and progressive muscle relaxation, which have demonstrated efficacy in both a clinical and non-clinical setting (for a review, see Keng, Smoski, \&Robins, 2011; Pennebaker \& Chung, 2012; Rupke, Blecke, \& Renfrow2006).

In addition, the program sought to educate participants about the negative consequences of employing emotional suppression as their main coping strategy (Bebko et al., 2011). The format of implementation chosen was a group setting based on the numerous benefits it provides, such as reach, scalability, and affordability. A meta-analysis (Sin \& Lyubomirsky, 2009) noted the strength of group therapy, stating that this method incurs strong benefits, second only to individualized training, which is more costly and time consuming. Relatedly, a recent study found support for the use of everyday settings, such as community centers, workplaces, and schools, noting that these interventions may reach those who would not use a conventional setting (Kazdin \& Rabbitt, 2013). Based on these factors, utilizing a group format in a naturalistic setting (university) was deemed the most effective methodology for the current research.

The present research assessed the effectiveness of the program in two stages. In the first stage, Study 1 , the program was developed andthe social validity was tested with a sample of graduate students. In the second stage, Study 2, the efficacy of the program was assessed withina community sample. It is hypothesized that the findings of the pre- sent study are beneficial to counselors by providing empirical research on the effectiveness of brief resilience training. This program may have a broader application, as it could be used by local mental healthcenters, employed by university counselors as group training, and utilized by high schools to cultivate emotional competence in lateadolescence.

\section{STUDY 1}

Method

Participants. A focus group of 10 students $(7$ females and 3 males)was drawn from the graduate department of psychology at a universityin London. No incentives were offered for participation. The meanage was 24.36 years $(S D=3.39)$, with a range from 22 to 28 years. Participants were administered the scales in a group sessions $(n=10)$. The program was implemented for 4 sessions, with each lasting between 1.5 and 2 hours. Data analysis criteria required each participant to attend at least 3 of the 4 workshops for data inclusion; the attrition rate was $17 \%$.

Instruments. A baseline questionnaire package including a consent form, a general information questionnaire, the Emotion Regulation Questionnaire (ERQ), and the Depression, Anxiety and Stress Scales (DASS-21) was used to collect data; questionnaires will be elaboratedupon in Study 1 , the primary focus of this article.

Treatment. The workshops covered a variety of topics, including identifying emotions, defining emotion regulation, differentiating between emotional reappraisal and suppression, learning to relax through progressive muscle relaxation, decreasing rumination through expressive writing, becoming engaged in the present moment through mindfulness, andregulating mood through positive self-talk. 
Table 1 includes the results from a Wilcoxon Signed Ranks Test that showed a significant decrease in the scores for negative affect from pre-intervention $(M=1.79, S D=0.73)$ to postintervention $(M=1.34, S D=0.51) ; Z(9)=2.67, p=0.01)$ for the expressive writing workshop.

\begin{tabular}{lll}
\hline Positive and Negative Affect Schedule (PANAS) Dimension & Z & $p$ \\
\hline EW Positive affect 1 \& EW Positive affect 2 & 1.47 & $\mathrm{~ns}$ \\
EW Negative affect 1 \& EW Negative affect 2 & 2.67 & 0.01 \\
ST Positive affect 1 \& ST Positive affect 2 & 2.20 & 0.03
\end{tabular}

Table 1. Wilcoxon Signed Ranks Test $(n$ $=10$ ) for Expressive Writing (EW) and Self-Talk (ST) Workshops

Table 2. Social Validity of Workshops $(n=10)$

\begin{tabular}{lll}
\hline Workshop & Benefit & Improvement \\
\hline Expressive writing & $8 / 10$ & $7 / 10$ \\
Self-talk & $8 / 10$ & $8 / 10$ \\
Mindfulness & $6 / 10$ & $5 / 10$ \\
Reappraisal & $6 / 10$ & $6 / 10$ \\
\hline
\end{tabular}

For the self-talk workshop, results showed a significant increase in the scores for positive affect from pre-intervention $(M=2.30, S D=0.87)$ to post-intervention $(M=2.73, S D=0.87) ; Z(6)=$ $2.20, p=0.03$. The Wilcoxon, with $p$-value of 0.03 , should be interpreted with caution, asit would be forfeited with a Bonferroni correction for type 1 error $(p<0.025)$.

This study assessed the social validity of the training program in order to evaluate participants' acceptance. Findings revealed that themajority of participants felt the workshops were significantly beneficial and saw substantial improvement in their skills across workshops (see Table 2). Qualitatively, in order to improve the program, participants recommended less academic terminology, a reduction in surveys, andan increase in activities and worksheets.

\section{Discussion}

The statistical findings revealed that the program significantly increased positive affect and decreased negative affect as the work- shops progressed for the self-talk and expressive writing workshop. Itwas an unexpected finding that participants would have increased positive affect after writing about stressful and negative life events; that does not align with general findings in the literature(Pennebaker \& Chung, 2012). However, the small sample size shouldbe taken into consideration when interpreting this finding. Overall, the majority of participants were satisfied with the programand reported improved emotion regulation competencies over the course of the training. In general, the expressive writing and self-talk workshops were considered more beneficial than the mindfulnessand reappraisal workshops, which coincides with the quantitative data (higher benefit and improvement scores). This finding may be due to the heavy reliance of these workshops on cognitive behavioral techniques, which are currently the gold standard in psychosocial 
interventions (Otte, 2011).

\section{STUDY 2}

Study 2 involved the implementation and validation of the training program developed in Study 1. Study 2 contained a psychoeducational component, application through directed exercise, and pre- post- andfollow-up measurements.

\section{Method}

Participants. A community sample of 75 participants was randomly allocated to either a treatment $(n=38)$ or a non-active control $(n=37)$ group. The sample consisted of 48 females and 27 males, with an average age of 36.68 years $(S D=14.90)$, ranging from 18 to 76 years old. Demographic data for each group was as follows: The control group consisted of 10 males $(27 \%)$ and 27 females $(73 \%)$; amean age of 37.65 ; and a predominantly Caucasian sample, $83.8 \%$. The treatment group consisted of $21(55.3 \%)$ females and 17 males $(44.7 \%)$; a mean age of 35.74 ; and a predominantly Caucasian sample, $89.5 \%$. The study attrition rate was $12 \%$.

Instruments. In the frame of the first study, Study 2 utilized the Emotion Regulation Questionnaire (ERQ), Depression, Anxiety and Stress Scales (DASS-21), and the Positive and Negative Affect Scale (PANAS). Additionally, the Satisfaction with Life Scale (SWLS) was added to the questionnaire package in Study 2 in order to have a positive, global measure of subjective well-being.

- $\quad$ Emotion Regulation Questionnaire (ERQ; Gross \& John, 2003) is a 10-item questionnaire measuring suppression (4 items) and reappraisal (6 items) on a 7-point Likert scale (1 = strongly disagree; 7 = strongly agree).

- Depression, Anxiety and Stress Scales (DASS-21; Norton, 2007) is a revised questionnaire measuring anxiety, depression, and stress (Lovibond \& Lovibond,1995). The scale is measured on a 4-point scale $(0=$ did not apply to me at all; $3=$ applied to me very much, or most of the time). The construct validity ofthis scale has been demonstrated in a large non-clinical sample (Henry \& Crawford, 2005).

- $\quad$ Positive and Negative Affect Scale (PANAS; Watson, Clark, \& Carey, 1988) is a 20-item questionnaire measuring state levels of positive and negative affect. It determines positive affect with 10 adjectives such as "excited" and "strong" and negative affect with 10 adjectives such as "hostile" and "jittery." This questionnaire is measured on a 5-point Likert scale ( 1 = veryslightly or not at all; $5=$ extremely).

- $\quad$ Satisfaction with Life Scale (SWL; Diener, Emmons, Larsen, \& Griffin, 1985) is a 5-item questionnaire measuring overall degree of life fulfillment. This scale is anchored on a 7-point Likert scale $(1$ = strongly disagree; 7 = strongly agree $)$.

Adequate reliability (Chronbach's alpha $>0.70$ ) scores were found for all measures utilized within this study.

Procedure. Initially, liability insurance was obtained and institutionalreview board (IRB) approval was garnered from a university in London.Participants were recruited from posters placed at a local university, on public message boards, and via recruitment from an undergraduate psychology class. No incentives were offered for participation. 
All participants were supplied with a questionnaire package containing a letter of intent, a consent form, a demographic questionnaire, the data collection instruments, and a debrief form in the initialinformational session. Following the initial session, participants in the treatment group were expected to attend a weekly 120-minute structured workshop over the next 4 weeks. Workshops were held in asmall group setting. Multiple workshops were held throughout the week to accommodate participants' schedules. All workshops were conducted by the same facilitator, the first author, to ensure consistency of material and presentation style.

Study 2 utilized a non-active control group. After the baseline questionnaire was completed at time 1, the control group remained inactive until the second baseline measurement was taken after the training program had been completed, at which time they completed the questionnaire package for the second time. Approximately oneyear after the intervention, a follow-up measurement was carried outvia email. A condensed follow-up, using an electronic questionnaire package containing the ERQ, SWL, and DASS, was distributed to all original participants, from both the intervention and the control group. At this stage, study attrition was $39 \%$.

\section{Treatment.}

- Workshop 1: Introduction to Emotion Regulation took an in-depth look at labeling and categorizing emotions on a behavioral, cognitive, and physio-logical level. The topic of emotion regulation was outlined and activitiescompleted to demonstrate the advantages of reappraisal when compared with suppression.

$\cdot$

- Workshop 2: Expressive Writing $(E W)$ is a journaling technique where one reflects on their deepest thoughts and feelings in relation to stressful or significant life events (Pennebaker \& Beall, 1986). The goal of the session was to decrease rumination in order to decrease depressive symptomologyvia the EW paradigm.

- Workshop 3: Mindfulness is non-elaborative awareness of present moment experience without judgment (Kabat-Zinn, 2003). The basic principles of mindfulness were explained, and worksheets and activities were completed.

- Workshop 4: Self-Talk and Cue-Controlled Progressive Muscle Relaxation. Conceptsof reappraisal and cognitive restructuring were revisited. The thought-stop-ping technique was outlined, and worksheets and activities were completed.The workshop finished by teaching diaphragmatic breathing, progressive muscle relaxation, and cue-controlled relaxation.

Following the framework of Study 1, the same format was used; however, workshops were modified based on qualitative feedback from Study 1.

After the intervention, participants were debriefed verbally and in writing. The debrief form discussed the nature of the study while reiterating confidentiality of information and right to withdraw.

Results

Table 3 shows descriptive statistics for all psychometric questionnaires utilized. In general, all constructs in the control group demonstrated no statistically significant mean shift. In contrast, 
within the treatment group, mean shifts were demonstrated within the expected directions. Specifically, life satisfaction and reappraisal increased, whereas depression symptomology, anxiety, and stress decreased. When comparisons are made across standard deviations, the data within the treatment group show narrower dispersion. See Table 4 for comparisons against population norms.

Table 3. Descriptive Statistics for Study Variables $(n=75)$

\begin{tabular}{|c|c|c|c|c|c|c|}
\hline \multirow[b]{2}{*}{ Control } & \multicolumn{2}{|c|}{ Pre- } & \multicolumn{2}{|c|}{ Post- } & \multicolumn{2}{|c|}{ Follow-up } \\
\hline & Mean & $S D$ & Mean & $S D$ & Mean & $S D$ \\
\hline Life satisfaction & 25.73 & 5.59 & 25.36 & 5.08 & 25.22 & 5.24 \\
\hline ERQ-Suppression & 3.43 & 0.77 & 3.41 & 0.82 & 3.20 & 1.02 \\
\hline ERQ-Reappraisal & 5.09 & 0.98 & 4.89 & 0.93 & 4.83 & 0.72 \\
\hline DASS-Stress & 10.88 & 9.32 & 11.09 & 8.28 & 11.04 & 7.91 \\
\hline DASS-Anxiety & 5.13 & 6.53 & 3.77 & 5.55 & 6.96 & 7.23 \\
\hline DASS-Depression & 6.75 & 7.07 & 5.83 & 6.94 & 7.65 & 7.90 \\
\hline \multicolumn{7}{|l|}{ Treatment } \\
\hline Life satisfaction & 22.76 & 8.35 & 23.05 & 7.50 & 25.78 & 6.16 \\
\hline ERQ-Suppression & 3.63 & 1.10 & 3.44 & 1.19 & 3.80 & 1.17 \\
\hline ERQ-Reappraisal & 4.83 & 1.30 & 5.20 & 1.27 & 5.60 & 0.91 \\
\hline DASS-Stress & 16.25 & 9.29 & 11.46 & 8.51 & 10.09 & 8.17 \\
\hline DASS-Anxiety & 7.42 & 7.63 & 4.95 & 5.07 & 2.96 & 4.55 \\
\hline DASS-Depression & 9.33 & 9.74 & 6.49 & 8.20 & 6.87 & 6.79 \\
\hline
\end{tabular}

At baseline, independent samples $t$-test showed no significant difference between any constructs measured, with the exception of SWL:control group $(M=25.73, S D=5.59)$, intervention group $(M=22.76, S D=8.35) ; t(73)=1.80, p=.008$.

A 2 (treatment and control group) $\times 3$ (pre-, post- and follow-up)repeated measure ANOVA showed significant interaction betweenreappraisal and the within-subject factor, $F(2,43)=$ $7.21, p=.002 . \eta 2=0.25$. Findings revealed that reappraisal increased over time in the treatment group. Post-hoc comparisons were conducted for all measurements. The results showed a significant difference in the mean scores of reappraisal $t(22)=3.20, p=.003$. Specifically, thetreatment group $(M=5.60, S D=0.91)$ had higher reappraisal scores than the control group $(M=4.83, S D=0.72)$. In addition, paired samples $t$-tests were run on the treatment group to compare reappraisal at different time points during the intervention. A significant increase in the mean scores of reappraisal were found between the pre- $(M=4.83$, $S D=1.30)$ and the follow-up measurement $(M=5.60, S D=0.91) ; t(22)=3.20, p=.003$. Further, a repeated measures ANOVA was run independently on the intervention group, using aBonferroni adjusted alpha level $.017(.05 / 3)$ per test, to determinewhether changes in reappraisal over time were significant. The ANOVA indicated a significant interaction between reappraisal and the within-subject factor $F(2,21)=5.12, p=.010, \eta 2=0.19$. The results from three paired samples $t$-tests, comparing the mean scores of reappraisal in the intervention group at pre-, post- and follow-up, revealed the mean scores of reappraisal between the pre- $(M=4.83, S D=1.30)$ and the follow-up measurement $(M=5.60, S D=0.91)$ weresignificantly different $(t(22)=3.20, p=.003)$.

Repeated measures ANOVAs run on anxiety, stress, depression, and satisfaction with life were nonsignificant; however, the within-subjects factor for anxiety showed a clear tendency towards significance $(p=0.05)$, and the between subjects factor for depression demonstrated anonsignificant trend toward significance $(p=0.10)$. 


\begin{tabular}{|c|c|c|c|c|c|c|c|c|}
\hline & & & & & \\
\hline & Pre- & Severity & Post- & Severity & Pre- & Severity & Post- & Severity \\
\hline Stress Normal & 16.25 & Mild & 10.09 & & 10.88 & Normal & 11.04 & \\
\hline Anxiety Normal & 7.42 & Mild (borderline) & 2.96 & & 5.13 & Normal & 6.96 & \\
\hline Depression Normal & 9.33 & Mild (borderline) & 6.87 & & 6.75 & Normal & 7.65 & \\
\hline Satisfaction with life & 22.76 & Average & 25.78 & High & 25.73 & High & 25.22 & High \\
\hline Reappraisal & 4.83 & Normal & 5.60 & High & 5.09 & High & 4.83 & Normal \\
\hline
\end{tabular}

Table 4. Paired Sample $t$-Test for Group Norms with Severity Level Against Population Norms

Treatment Control

\section{Discussion}

In support of the primary hypothesis of Study 2, analysis showed there was a significant interaction between reappraisal and the within-subject factor. In the treatment group, reappraisal increased significantly a year after the intervention occurred, while in the control group it decreased significantly. However, follow-up comparisons of between-group differences at the pre- and post-measurement were not significantly different. This finding may indicate thatit may have taken more time for group differences to reach significance. The general trend in each group shows reappraisal was gradually declining in the control group and significantly improving within the treatment group. Thus, an additional follow-up is war- ranted. In mental health research, it is common to do long-term follow-ups 10 to 15 years later in order to see if the intervention had sustaining effects (Jonsson, et al., 2011). The increase in reappraisalis particularly significant when considering the burgeoning literature demonstrating that positive interventions successfully prevent psychopathology and may be a key component of best practice psychotherapy.

In support, a study by Duckworth and colleagues (2005) determined the frequent use of reappraisal significantly distinguished a non-clinical from a clinical sample that were more likely to employ maladaptive strategies (self-blame and catastrophizing). They recommend targeting cognitive emotion-regulation strategies for both prevention and clinical intervention. This opinion is further supported by recent research demonstrating the efficacy of group emotion-regulation interventions in at-risk and clinical populations (Cameron \& Jago, 2008; Smyth \& Arigo, 2009). Similarly, a recent meta-analysis by Siegenthaler, Munder, and Egger (2012)determined a 40\% reduced risk of psychopathology for childrenof parents with mental illness who had undergone preventative interventions. Relatedly, Greenberg (2008) argues for integrating an emotion-focused approach into standard cognitive behavioral therapy, since affect may be subject to different change principles than cognitions, as emotions are not controlled by logical reasoning; change on the cognitive-rationalintellectual level may not always change the emotional meaning of the situation. Thus, findings from the current research, when taken in the context of the aforementioned literature, suggested a need for future research to test the intervention in at-risk and clinical samples.

Although the current study garnered promising initial findings, limitations require discussion. During statistical analysis, within the treatment group, a substantial amount of the data was trending in the right direction but was just below the acceptable cut-off for statisticalsignificance. In addition, standard deviations for the majority of surveys were very large. Thus, a methodological weakness was the lack ofstatistical power, which in part may be due to small 
sample sizes. However, to determine significant statistical power for the efficacy ofa universal mental health promotion program would require a large- scale study, with treatment and control groups each in the tens of thousands, which was beyond the scope of the current research. A final limitation was the use of a non-active (untreated comparison) control group. It is possible that the positive intervention effectsoccurred due to a placebo effect arising from group participation ingeneral, rather than from the specific techniques taught during the intervention itself. This limitation can be addressed in future researchby employing an active control group trained in an unrelated activity,such as academic motivation. However, it is important to note that two psychosocial intervention studies enhancing adult emotional competencies (Kotsou et al., 2011; Nelis et al., 2011) addressed this issue. Intheir first study, a non-active control group was utilized, while in theirsecond study, an active control group was employed. Results from both studies garnered comparable results.

\section{CONCLUSION}

Although many types of psychological interventions exist, the intervention used in the current study is unique because it was resiliencytraining designed for an adult community-based sample that was grounded in theory-based techniques. This intervention attemptedto provide participants with an adaptive "emotion regulation toolkit" for combating daily life stressors.

To paraphrase Albert Einstein, "Science and society cannot solve the mental health problems of today by using the same kind of thinking that was used before when. . science and society helped create them" (Keyes, 2007, p. 106). Ultimately, a paradigm shift isneeded, moving away from a reactive healthcare approach to a more proactive, holistic model. Within the healthcare field, we need to redefine and address the importance of mental well-being, viewing it as a multifaceted construct involving both the absence of psycho- pathology and the presence of positive psychological assets (Sin \& Lyubomirsky, 2009). The results of this research may provide the firststep in this direction, by demonstrating that a brief, theoretically derived intervention can elicit enduring, positive changes in mental health. These findings may have broader implications for clinicians and researchers; thus, further replication is warranted in both clinical and non-clinical settings to test the limits of the intervention's efficacy. These initial findings are promising and may have application in the health sector due to the brevity and positive outcome of this low-cost,preventative intervention; thus, further exploration is warranted.

\section{REFERENCES}

Baer, R. A. (2003). Mindfulness training as a clinical intervention: A conceptual and empirical review. Clinical Psychology: Science and Practice, 10,125-143.

Bebko, G. M., Franconeri, S. L., Ochsner, K. N., \& Chiao, J. Y. (2011). Look before you regulate: Differential perceptual strategies underlying expressive suppression and cognitive reappraisal. Emotion, 11, 732-742.

Bolier, L., Haverman, M., Westerhof, G. J., Riper, H., Smit, F., \& Bohlmeijer, E. (2013). Positive psychology interventions: A meta-analysis of randomized controlled studies. BMC Public Health, 13(1), 119. 
Cameron, L. D., \& Jago, L. (2008). Emotion regulation interventions: A common-sense model approach. British Journal of Health Psychology, 13 (2), 215-221.

Cisler, J. M., \& Olatunji, B. O. (2012). Emotion regulation and anxiety disorders. Current Psychiatry Reports, 14, 182-187.

Cuijpers P., Munoz R. F., Clarke G. N., \& Lewinsohn P. M. (2009). Psychoeducational treatment and prevention of depression: The coping with depression course thirty years later. Clinical Psychological Review, 29, 449-458.

Diener, E., Emmons, R., Larsen, J., \& Griffin, S. (1985). The Satisfaction with Life Scale. Journal of Personality Assessment, 49, 71-75.

Duckworth, A. L., Steen, T. A., \& Seligman, M. E. P. (2005). Positive psychology in clinical practice. Annual Review of Clinical Psychology, 1, 629-651.

Fledderus M., Bohlmeijer E., Smit F., \& Westerhof G. J. (2011). Mental health promotion as a new goal in public mental health care: A randomized controlled trial of an intervention enhancing psychological flexibility. American Journal of Mental Health, 100, 2372-2378.

Greenberg, L. (2008). Emotion and cognition in psychotherapy: The transforming power of affect. Canadian Psychology/Psychologie Canadienne, 49(1), 49.

Gross, J. J. (1998). Antecedent- and response-focused emotion regulation: Divergent consequences for experience, expression, and physiology. Journal of Personality and Social Psychology, 74, 224237.

Gross, J. J. (2013). Emotion regulation: Taking stock and moving forward.

Emotion, 13(3), 359.

Gross, J. J., \& John, O. P. (2003). Individual differences in two emotion regulation processes: Implications for affect, relationships, and well- being. Journal of Personality and Social Psychology, $85,348-362$.

Gross, J. J., \& Levenson, R. W. (1997). Hiding feelings: The acute effects of inhibiting negative and positive emotion. Journal of Abnormal Psychology,106, 95-103.

Henry, J. D., \& Crawford, J. R. (2005). The short-form version of the Depression Anxiety Stress Scales (DASS-21): Construct validity and normative data in a large non-clinical sample. British Journal of Clinical Psychology, 44(2), 227-239.

John, O. P., \& Gross, J. J. (2004). Healthy and unhealthy emotion regulation: Personality processes, individual differences, and life span development.Journal of Personality, 72(6), 1301-1334.

Jonsson, U., Bohman, H., von Knorring, L., Olsson, G., Paaren, A., \& von Knorring, A. L. (2011). Mental health outcome of long-term and episodic adolescent depression: 15-year follow-up of a community sample. Journal of Affective Disorders, 130, 395-404.

Joormann, I. H., \& Gotlib, J. (2010). Cognition and depression: Current status and future directions. Annual Review of Clinical Psychology, 6, 285-312.

Kabat-Zinn, J. (2003). Mindfulness-based interventions in context: Past, present, and future. Clinical Psychology: Science and Practice, 10(2), 144-156. 
Kazdin, A. E., \& Rabbitt, S. M. (2013). Novel models for delivering mentalhealth services and reducing the burdens of mental illness. Clinical Psychological Science, 2167702612463566.

Keng, S. L., Smoski, M. J., \& Robins, C. J. (2011). Effects of mindfulness onpsychological health: A review of empirical studies. Clinical Psychology Review, 31(6), 1041-1056.

Keyes, C. L. (2007). Promoting and protecting mental health as flourishing: A complementary strategy for improving national mental health.American Psychologist, 62, 95-108.

Koole, S. L. (2009). The psychology of emotion regulation: An integrativereview. Cognition and Emotion, 23, 4-41.

Kotsou, I., Nelis, D., Gregoire, J., \& Mikolajczak, M. (2011). Emotional plasticity: Conditions and effects of improving emotional competencein adulthood. Journal of Applied Psychology, 96, 827839.

Lovibond, P. F., \& Lovibond, S. H. (1995). The structure of negative emotional states: Comparison of the Depression Anxiety Stress Scales (DASS) with the Beck Depression and Anxiety Inventories.

Behaviour Research and Therapy, 33, 335-343.

McLaughlin, K. A., \& Hatzenbuehler, M. L. (2009). Mechanisms linking stressful life events and mental health problems in a prospective, com- munity-based sample of adolescents. Journal of Adolescent Health, 44(2), 153-160.

Meulenbeek P., Willemse G., Smit F., van Balkom A., Spinhoven P., \& Cuijpers P. (2010). Early intervention in panic: Pragmatic randomized controlled trial. British Journal of Psychiatry, 196, 326331.

Nelis, D., Kotsou, I., Quoidbach, J., Hansenne, M., Weytens, F., Dupuis, P., \& Mikolajczak, M. (2011). Increasing emotional competence improves psychological and physical wellbeing, social relationships, and employability. Emotion, 11, 354-366.

Norton, P. J. (2007). Depression Anxiety and Stress Scales (DASS): Psychometric analysis across four racial groups. Anxiety, Stress, and Coping: An International Journal, 20, 253-265.

Otte, C. (2011). Cognitive behavioral therapy in anxiety disorders: Current state of the evidence. Dialogues Clinical Neuroscience, 13(4), 413-421.

Pennebaker, J. W., \& Beall, S. K. (1986). Confronting a traumatic event:Towards an understanding of inhibition and disease. Journal of Abnormal Psychology, 95, 274-281.

Pennebaker, J. W., \& Chung, C. K. (2012). Expressive writing and its links tomental and physical health. In H. S. Friedman (Ed.), Oxford handbook ofhealth psychology. New York, NY: Oxford University Press.

Rupke, S. J., Blecke, D., \& Renfrow, M. (2006). Cognitive therapy for depression. American Family Physician, 73(1), 83-86.

Siegenthaler, E., Munder, T., \& Egger, M. (2012). Effect of preventive interventions in mentally ill parents on the mental health of the offspring:Systematic review and meta-analysis. Journal of the American Academy of Child \& Adolescent Psychiatry, 51(1), 8-17.

Sin, N. L., \& Lyubomirsky, S. (2009). Enhancing well-being and alleviating depressive symptoms with positive psychology interventions: A practice-friendly meta-analysis. Journal of Clinical Psychology, 65(5), 467-487. 
Smyth, J. M., \& Arigo, D. (2009). Recent evidence supports emotion-regulation interventions for improving health in at-risk and clinical populations. Current Opinion in Psychiatry, 22(2), 205-210.

Watson, D., Clark, L. A., \& Carey, G. (1988). Positive and negative affectivityand their relation to anxiety and depressive disorders. Journal of Abnormal Psychology, 97, 346-353. 